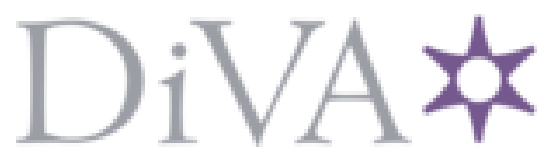

http://www.diva-portal.org

Postprint

This is the accepted version of a paper presented at 2O2O IEEE 31st Annual International Symposium on Personal, Indoor and Mobile Radio Communications.

Citation for the original published paper:

Masoudi, M., Khagagy, M G., Soroush, E., Giacomelli, D., Morosi, S. et al. (2020)

Reinforcement Learning for Traffic-Adaptive Sleep Mode Management in 5G Networks In:

N.B. When citing this work, cite the original published paper.

Permanent link to this version:

http://urn.kb.se/resolve?urn=urn:nbn:se:kth:diva-278590 


\title{
Reinforcement Learning for Traffic-Adaptive Sleep Mode Management in 5G Networks
}

\author{
Meysam Masoudi *, Mohammad Galal Khafagy * ॥, Ebrahim Soroush ${ }^{\dagger}$, \\ Daniele Giacomelli ${ }^{\S}$, Simone Morosi ${ }^{\S}$, and Cicek Cavdar * \\ * KTH Royal Institute of Technology, Sweden. $\|$ The American University in Cairo (AUC), Egypt. \\ $\S$ University of Florence, Italy. ${ }^{\dagger}$ Zi-tel Company, Iran. \\ E-mail: \{masoudi, khafagy, cavdar\}@kth.se, ebrahim.soroush@gmail.com, \{dani.giacomelli, simone.morosi\}@unifi.it
}

\begin{abstract}
The ever-increasing energy consumption of mobile networks has concerned operators about their growing network running costs. Among the different segments of mobile networks, base stations (BSs) have a major energy consumption share. To reduce BS consumption, BS components with similar (de)activation times can be grouped and put into sleep during their times of inactivity. The deeper and more energy-efficient a sleep mode (SM) is, the longer (de)activation time it takes to transition, which incurs a proportional service interruption. Therefore, it is challenging to timely decide on the best SM, bearing in mind the daily traffic fluctuation and imposed service level constraints on delay/dropping. In this study, we leverage an online reinforcement learning technique, i.e., SARSA, and propose an algorithm to opt SM given time and BS load. We use real mobile traffic obtained from a BS in Stockholm to evaluate the performance of the proposed algorithm. Simulation results show that considerable energy saving can be achieved at the cost of acceptable packet dropping level compared to two lower/upper baselines, namely, fixed (non-adaptive) SMs and optimal non-causal solution.
\end{abstract}

Keywords-5G, base station sleeping, discontinuous transmission, energy efficiency, reinforcement learning.

\section{INTRODUCTION}

Cellular network traffic is exponentially increasing together with the growing number of services offered by the fifth generation of mobile networks (5G). Mobile operators anticipate a sharp surge in network energy consumption due to such a growing load, placing network energy efficiency as one key objective in current and future deployments [1], [2]. Studies show that more than $80 \%$ of the overall network energy consumption take place within base stations (BSs) [3]. In order to maintain the operational sustainability of such networks, it is crucial to devise efficient means for BS energy minimization while avoiding any adverse impact on the offered quality-of-service (QoS) to served users, e.g., dropping rate for data services. Recently in [4], it is shown that BS transceiver chain components can be divided according to their (de)activation time scale into 4 groups. Each group of components (with similar transition times) can be simultaneously put to sleep for a certain time duration, defined as sleep modes (SMs). Implementing SMs can save BS energy at the cost of bearing some 0service interruption. This interruption, e.g., delay/dropping, may have more severe impact during daytime when network traffic congestion usually increases. Therefore, meticulous SM implementation is required to mitigate QoS degradation. In this study, we propose an online traffic-adaptive BS energy saving algorithm that utilizes SMs while considering service impact in terms of dropping.

Among SMs, the most shallow is known as cell DTX, in the order of one symbol time, where the power amplifier

978-1-5386-8110-7/19/\$31.00 (c) 2019 IEEE
(PA) is opportunistically shutdown in times of inactivity. The authors in [5] study cell DTX in heterogeneous networks and investigate the impact of BS density and traffic load on potential energy savings. The study in [6] aims at loadadaptive SM management considering deep, long-term SMs subject to QoS guarantee. In [7], the ON-OFF state of 4G BSs is evaluated, quantifying the impact of BS sleep on the QoS, e.g., dropping/delay. In [8], the authors evaluate the ON-OFF state of mm-wave small cells, considering cellular traffic data from Tokyo. One of the enablers for $5 \mathrm{G}$ is cloud radio access networks (C-RAN) and its variants. Although C-RAN is intrinsically saving energy by centralization of baseband units, this architecture puts pressure over transport and cloud network resources. [9], [10] analyze the trade-off between energy saving and resource consumption in hybrid C-RAN considering ON-OFF sleep option for processing resources.

When multiple SMs are allowed, a timely best SM selection policy becomes a vital part of the saving process. In [4], unlike the previous work with a binary state, a BS power consumption model is proposed with four BS SMs, each with different minimum transition duration. BSs can activate one of the SMs depending on the duration of BS idle time, i.e., duration in which no service is requested. Among these SMs, deeper SMs longer than 1 frame cannot be implemented in $4 \mathrm{G}$ BSs due to limitation of $4 \mathrm{G}$ periodic signaling, which is transmitted every $1 \mathrm{msec}$. In order to better leverage the SMs, the standardization effort in [11] proposes 5G BSs to extend the period of signaling up to $160 \mathrm{msec}$ and hence deeper and longer SMs can be accommodated. The authors in [12][14] investigate these 4 SM implementations. They assume gradual (de)activation of SMs and decide on the duration of each SM. In [12] an algorithm is proposed to implement the SMs considering periodic signaling bursts. In [13], an SM management solution is studied based on Q-learning approach to find the optimal duration for each SM level according to the load. In [14], the authors propose a trafficaware strategy to build a codebook for mapping the traffic load to the possible actions using Q-learning algorithm. The study in [15] proposes a BS sleeping approach and predicts the BSs inactivity time. Such a prediction enables selecting the best SM strategy while minimizing the adverse impact of BS activation/reactivation time on the serving latency. This study proposes control/data plane separation in 5G BSs which allows SM4 implementation (the deepest/longest SM). SM4 is also possible in co-coverage scenarios when basic coverage cells may carry all periodic signalling, leaving capacity-enhancing ones with better ability to sleep and save energy.

Pre-defining a certain order of SMs [12]-[14] may, however, return sub-optimal results. There exists an incentive to directly go from active mode, or shallow SMs, to the 
deepest possible mode to attain the best power saving without unnecessarily wasting time in intermediate levels. Going to the deepest SM may yield unbearable service delay or high packet dropping rate, while sticking with choosing the shallowest SM may result in missing the opportunity of energy saving. Therefore, it is not clear which SM is the best action to take, given the current load and state of the BS. In this paper, we aim at devising an adaptive algorithm that can dynamically take transition decisions based on the long-/short-term traffic load. We model the BS sleeping problem as a sequential Markov decision process (MDP), under uncertainty and restricted state-action sets. We utilize on-policy SARSA [16], a well-known reinforcement learning (RL) algorithm, to solve the MDP and track the load changes. We feed a pre-trained state-action transition table to the online system in its initialization phase and a periodic adaptation (or based on a trigger of a statistics change metric), the system can learn and modify the table on time. We apply the devised solution on a real traffic data set obtained from an operator's BS in Stockholm.

The rest of the paper is organized as follows. In Section II we present the investigated system model. In Section III, we design states, actions, reward function, and RL algorithm to solve the problem. We discuss and conclude the results in Sections IV and V.

\section{SYSTEM MODEL}

\section{A. Studied Network Scenario and Sleeping Modes}

In this work, we consider a co-coverage scenario where a BS site has two serving cells with overlapping coverage regions; a basic coverage cell and a capacity cell, each having a different frequency band (not interfering on each other). The capacity cell coverage area can be the entire or a partial subset of the basic coverage area, like in a heterogeneous network (HetNet) scenario, and we only focus here on the traffic in the overlapping region. The objective is to devise a load-adaptive algorithm that puts the capacity cell to sleep, by timely deciding on the best SM for energy saving while abiding by the packet drop rate specified in the mobile operator's license. According to [4, and the references therein], the BS hardware components can be grouped according to their activation/deactivation delays into 4 groups that can be shut down together to save power whenever they are not needed, forming 4 different levels of SMs.

1) SM1: This SM is the fastest, and the most shallow, whose duration is comparable to the symbol time $T_{\mathrm{s}}$. Whenever a BS does not have any traffic over the entire band of sub-carriers during symbol time per antenna, the PA can be switched off to save power. This mode is available and known in current technologies as microscale discontinuous transmission ( $\mu \mathrm{DTX})$.

2) SM2: A slightly deeper sleep level can be reached by switching off one more set of components with slower (de)activation transition time than that of SM1, if this longer delay can be afforded by the system. In this mode, the transition is on the transmission time interval (TTI) scale, i.e., $1 \mathrm{msec}$ (a duration of a sub-frame constituting 2 resource blocks (RBs) or 14 resource elements (REs) assuming frequency division duplexing (FDD) Frame Structure 1 [17])

3) SM3: This mode has a deeper sleeping level but with a minimum duration of a $10 \mathrm{msec}$ frame (10 sub-frames).
TABLE I: Power and Minimum Durations from [4], [18]

\begin{tabular}{|c|c|c|c|c|c|c|}
\hline \multirow[t]{2}{*}{ Mode } & \multicolumn{2}{|c|}{ Active } & \multirow{2}{*}{ SM1 } & \multirow{2}{*}{ SM2 } & \multirow{2}{*}{ SM3 } & \multirow{2}{*}{ SM4 } \\
\hline & $100 \%$ & $0 \%$ & & & & \\
\hline Pow. (W) & 55.7 & 15.2 & 12.2 & 0.754 & 0.648 & 0.471 \\
\hline Dur. (sec) & \multicolumn{3}{|c|}{$\frac{1}{T_{\mathrm{s}}: \text { fast modes }(\mathrm{FMs})}$} & $1 \mathrm{msec}$ & $10 \mathrm{msec}$ & $1 \mathrm{sec}$ \\
\hline
\end{tabular}

4) SM4: This is the deepest SM with a minimum duration of 100 frames $(1 \mathrm{sec})$.

In order to communicate with users, BSs have to periodically send signaling bursts. In LTE BSs, the following control signals are transmitted periodically: 1) Cellspecific Reference Signals (CRS), 2) Primary and Secondary Synchronization Signals (PSS and SSS, respectively), 3) Physical Broadcast CHannel (PBCH). These signals are very frequent, e.g. PSS and SSS are transmitted in the first and fifth LTE sub-frames. Therefore, it is not possible to benefit from SMs deeper than SM1. In 5G New Radio (5G $\mathrm{NR}$ ), operators are allowed to adjust their periodicity of synchronization signals to $5,10,20,40,80$ and $160 \mathrm{msec}$ [14]. Hence, the implementation of deeper SMs becomes feasible.

In this work, we seek to devise an adaptive system that can make the capacity cell dynamically hop from the active state to any of the SMs or back based on the instantaneous traffic load without any pre-dictated chronological order of modes or non-causal knowledge of future traffic. The basic coverage cell is assumed to hold all frequent periodic signalling on behalf of its underlying capacity cell(s), giving more flexibility for capacity cell(s) to go into deeper SMs without affecting the QoS at lower loads. The capacity cell will be only responsible for the control signalling associated with data transmission when it is active. Also, this implicitly restricts the depth of sleep for the basic coverage cell. As mentioned, this setting is particularly interesting and promising for HetNet scenarios where a central and wider basic coverage macro-cell overlaps with multiple cocoverage capacity cells with narrower coverage areas. In this setting, all co-coverage capacity cells can enjoy the freedom to go into deeper SMs, while the central cell holds all necessary periodic control signalling. Specifically, when separation of data and control signalling planes is readily implemented, data BSs (for capacity) can focus only on data traffic, while signalling BSs handle user connectivity issues [4].

\section{B. Adopted Power Consumption and Sleep Duration Model}

We adopt the BS power consumption model developed in [19], reported in [4, and the references therein], and demonstrated via an online tool by imec in [18]. The power consumption values in Table I are used throughout the numerical results section, which are obtained from the online tool in [18] using the configuration parameters summarized in Table II. We divide the six available operational modes shown in Table I (active (from $0+$ to $100 \%$ loaded), idle ( $0 \%$ loaded), and four SMs) into fast and slow mode groups. The fast mode (FM) group includes the modes in which the system can take an optimal decision on the spot for power consumption level and accommodated traffic based on the arriving traffic. Therefore, this mode includes all the three fast operational modes of duration $T_{\mathrm{s}}$; active, idle, and SM1 as shown in Table I. When the system is in this FM mode group, it can freely and instantaneously hop between the three member modes with direct function mapping from load 
to power consumption without any need for optimization. The slow mode group constitutes the remaining three modes which incur a transition delay from the time a decision is taken till it is in action. Such a delay will cause dropping or additional queuing delay of traffic arriving in the transition period and hence influences the overall system energy consumption and QoS performance.

In this work, we need to distinguish between deactivation time, reactivation time, and the variable sleep duration in between. The model in [4], however, only specifies the total deactivation/reactivation transition duration. We assume that when the system takes an SM decision it goes to sleep at once at the beginning of the symbol time and when a decision is taken to depart any of the SMs, the entire transition duration needs to pass first. For SM1, both deactivation and reactivation times are considered negligible. The sleep and reactivation power consumption levels for these SMs are considered equal, and hold the values in Table I.

\section{Traffic Model}

1) Operator Data Set: In the following numerical results, we use a data set from a Swedish mobile network operator. The data set only includes anonymized instantaneous data rates for aggregated uplink and downlink traffic. Each data set is covering a duration of two months in 2018, with one sample taken every 5 minutes. We focus on the aggregated downlink traffic of one of such BSs in one location in Stockholm, only considering the FDD downlink band for simplicity, and without loss of generality. Very similar studies can be performed to simultaneously take into account all different BS bands, considering both uplink and downlink. This coarse-grained original data set is processed as follows to obtain the desired finer traffic granularity as an input to the algorithm:

1) The instantaneous rate (in bits/sec) data set is converted into a value of instantaneous load (in RBs/symbol time) using the following direct calculation:

$$
\operatorname{load}=\frac{\text { rate }(\text { in bits } / \mathrm{sec}) \times T_{s}}{\log _{2}(M) \times 12} .
$$

The load is in (RBs/symbol time). The division by 12 in Eq. (1) is due to the fact that each RB spans 12 subcarriers, and hence includes 12 symbols (REs). A fixed $M$-QAM modulation scheme is assumed with $M=16$.

2) Since all days possess a similar load pattern along each day, we first interlace the samples during the available two months into a single day to have an original data set with slightly finer granularity.

3) Next, to obtain the data set with the desired fine granularity (one sample every symbol time, i.e., approximately every $72 \mu \mathrm{sec}$ ), we do the following.

a) An hourly histogram is generated for the data set.

b) All missing fine points within each hour are generated according to the hourly histogram.

\section{TRAFFIC-AWARE SEQUENTIAL DECISION MAKING FOR ENERGY MINIMIZATION UNDER UNCERTAINTY}

\section{A. System States and Actions}

1) Load-Aware System: A system operational state at time slot $i$, denoted as $s_{i}$, can only take one of the values from the set $\mathcal{S}$. The state is composed of current load status and the previously taken action, which in turn defines the current sleep status. The current load is defined as the number of users during the previous actions and is categorized into two levels; namely, i) Low when there is no user requests, and ii) High when there is at least one user request in the system. In transition periods, the system is said to be in the last state till the new state is in action. Also, a universal action set is defined in terms of 4 possible actions defined in $\mathcal{A}$ where here FM and SMk, with $k \in\{2,3,4\}$, denote the go-to-fast-mode and go-to-SM $k$ state transition actions, respectively.

$$
\begin{aligned}
\mathcal{S} & =\mathcal{A} \times \mathcal{L} \\
\mathcal{L} & =\{\text { Low, High }\} \\
\mathcal{A} & =\{\text { FM, SM2, SM3, SM4 }\}
\end{aligned}
$$

The fast mode includes all the micro-scale active, idle and SM1 states. The system environment information at time slot $i$ is fully captured via the following 5-tuple:

$$
\boldsymbol{e}_{i}=\left(p_{i}^{(\mathrm{b})}, l_{i}^{(\mathrm{b})}, p_{i}^{(\mathrm{c})}, l_{i}^{(\mathrm{c})}, l_{i}\right)
$$

where $p_{i}^{(\mathrm{b})}$ and $p_{i}^{(\mathrm{c})}$ are the basic and capacity cell power consumption, $l_{i}^{(\mathrm{b})}$ and $l_{i}^{(\mathrm{c})}$ represent the basic and capacity cell accommodated load in RBs, while $l_{i}$ is the total traffic load that needs to be served at the $i^{\text {th }}$ time slot, respectively. That is $l_{i}=l_{i}^{(\mathrm{b})}+l_{i}^{(\mathrm{c})}+d_{i}$ where $d_{i}$ represents the number of dropped RBs at the $i^{\text {th }}$ symbol time, which is the difference between the arriving traffic and total served traffic. We also define the total power consumption at the $i^{\text {th }}$ symbol time as $p_{i}=p_{i}^{(\mathrm{b})}+p_{i}^{(\mathrm{c})}$.

\section{B. Reward/Penalty Function Design}

Before designing the reward function, the following assumptions should be taken into consideration.

1) Power Saving Reward: While the capacity cell is operating in FM, we know that no dropping can happen. A power saving opportunity can be missed though if the system is operating in a shallow SM while a deeper one is possible. Taking capacity cell power consumption at SM1 $p_{\mathrm{SM} 1}^{(\mathrm{c})}$ as a reference value, a power saving metric at the $i^{\text {th }}$ time slot can be calculated as

$$
\tilde{r}_{i, p}=\frac{\left(p_{\mathrm{SM} 1}^{(\mathrm{c})}-p_{i}^{(\mathrm{c})}\right)^{+}}{p_{\mathrm{SM} 1}^{(\mathrm{c})}},
$$

where $(x)^{+}$is an operation that takes the value of $x$ when it is non-negative, while assigning a value of zero otherwise. With this definition, $\tilde{r}_{i, p}$ may take one of four possible values, corresponding to the 4 possible SMs, from 0 to $\tilde{r}_{p}^{\max }$, where $\tilde{r}_{p}^{\max }=\frac{\left(p_{\mathrm{SM} 1}^{(\mathrm{c})}-p_{\mathrm{S} 44}^{(\mathrm{c})}\right)}{p_{\mathrm{SM} 1}^{(\mathrm{c}}}$. We call the following normalized version, $r_{i, p}$, as the power saving reward: $r_{i, p}=\frac{\tilde{r}_{i, p}}{\tilde{r}_{p}^{\max }}$, where $r_{i, p} \in[0,1]$.

2) Dropping Penalty/Reward: When dropping happens while the system is in one of the deep SMs, i.e., $d_{i} \neq 0$, or in the case when $d_{i}=l_{i}^{(\mathrm{c})}=0$, the normalized dropping penalty can be calculated as $r_{i, d}=-\frac{d_{i}}{l_{\max }^{(\mathrm{c})}}$, which linearly scales with dropping and takes minimum and maximum values of 0 when $d_{i}=0$, and 1 when $d_{i}=l_{\max }^{(\mathrm{c})}$, respectively. When the system is in FM with $l_{i}^{(\mathrm{c})} \neq 0$, although no power saving is attained, there should exist a reward for the avoided 
dropping. That is, when $\left(d_{i}=0\right) \&\left(l_{i}^{(\mathrm{c})} \neq 0\right)$, the reward is $r_{i, d}=\frac{l_{i}^{(\mathrm{c})}}{l_{\max }^{(\mathrm{c})}}$. It hence follows that a suitable dropping reward function can be given as

$$
r_{i, d}= \begin{cases}\frac{l_{i}^{(\mathrm{c})}}{l_{\max }^{(\mathrm{c})}}, & \text { if }\left(d_{i}=0\right) \&\left(l_{i}^{(\mathrm{c})} \neq 0\right), \\ -\frac{d_{i}}{l_{\max }^{(\mathrm{c})}}, & \text { otherwise. }\end{cases}
$$

After the previous highlights, the incurred reward function at the end of the $i^{\text {th }}$ symbol time due to the action taken at the end of symbol time $i-1$ can now be plausibly defined as

$$
r_{i}=\alpha r_{i, p}+(1-\alpha) r_{i, d}
$$

where $\alpha$ is a weight parameter that can be set to give more emphasis to power saving or immunity against dropping.

\section{Reward Calculation, Learning, and Action Times}

When an action is taken at the $i^{\text {th }}$ symbol time to depart state $s_{i}$, all symbol times with indices $\{j\}_{j=i+1}^{i+w_{s_{i}-1}}$ will be locked to the same state $s_{i}$ and no further action can be taken until transition period fully elapses. Hence, rewards/penalties incurred in it should be designed to return on the state-action pair $\left(s_{\text {prev }}, a_{\text {prev }}\right)$. Formally, at the end of any non-frozen symbol time $i$, where the system is in state $s_{i} \in \mathcal{S}$, it is required to take an action $a_{i} \in \mathcal{A}$ to move to either

1) the same state $s_{i+1}=s_{i}$ at the following symbol time $i+1$ when $\left(l_{i}, a_{i}\right)=s_{i}$, or

2) a state $s_{i+w_{s_{i}}}$ when $a_{i} \neq a_{i-1}$ along symbol time $i+$ $w_{s_{i}}$ or $l_{i} \neq l_{i-1}$, with $w_{s_{i}}$ being the transition duration from state $s_{i}$ in symbol times as also included in Table I as $\boldsymbol{w}=\left(w_{1}, w_{2}, w_{3}, w_{4}\right)=(1,14,140,14000)$. We assume that if the BS is activated, it should be in FM mode (active mode or only SM1) for 14 symbol times. This will help the BS to avoid ping-pong effect in the burst arrivals [7].

Only at the end of such transition periods the system can calculate the reward of its previous transition action. When learning is applied, these are also the times where the system should learn and update its state-action table. We hence generalize the simple $i^{\text {th }}$ action reward function in Eq. (5) to be an average over this extended period of time as

$$
\bar{r}_{i}=\frac{\sum_{j=i-w_{k}-1}^{i}\left(\alpha r_{j, p}-(1-\alpha) r_{j, d}\right)}{w_{k}},
$$

which is calculated at the end of time slot $i$.

\section{Proposed Solution Algorithm}

Before proceeding with the proposed solution, the 5tuple state in Eq. (2), and accordingly its space, can be significantly shrunk using the following deductions. Since the basic coverage cell is allowed only to be active or go into SM1, the basic cell's power consumption $p_{i}^{(\mathrm{b})}$ is a direct function of its load $l_{i}^{(\mathrm{b})}$ given the power consumption model. Also, due to the affine power consumption with a constant positive-valued power consumption component at zero load, as demonstrated through the available models in the literature, it is always optimal to first fill up the basic coverage resources before activating the capacity cell, i.e., the optimal pair $\left(l_{i}^{(\mathrm{b})}, l_{i}^{(\mathrm{c})}\right)$ given $l_{i}$ from a power consumption perspective will always return a zero-valued $l_{i}^{(\mathrm{c})}$ unless $l_{i}^{(\mathrm{b})}$

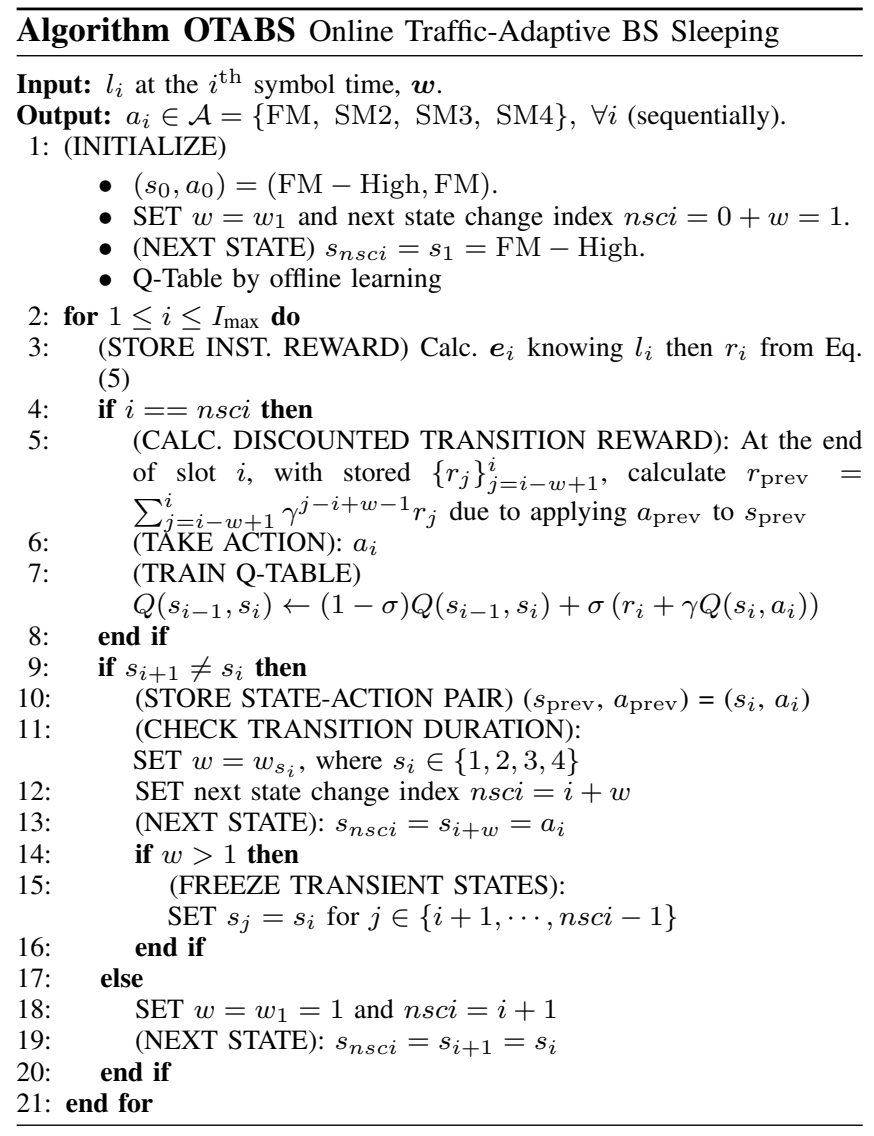

reaches $l_{\max }^{(\mathrm{b})}$, that is $l_{i} \geq l_{\max }^{(\mathrm{b})}$. Hence, given $l_{i}$, the optimal $l_{i}^{(\mathrm{b})}$ and $p_{i}^{(\mathrm{b})}$ values can be directly computed. There is hence no need to base our actions on them. The accommodated load at the capacity cell, $l_{i}^{(\mathrm{c})}$, however, depends on the sleeping mode and the difference between the instantaneous load and the accommodated load in the basic cell. Based on the previous explanation, the following system state is sufficient to capture the reduced tuple $\tilde{\boldsymbol{e}}_{i}=\left(p_{i}^{(\mathrm{c})}, l_{i}\right)$. Given $p_{i}^{(\mathrm{c})}$, we can know the sleeping mode of the capacity cell, and its supported load $l_{i}^{(\mathrm{c})}$. Knowing the capacity cell operational/sleep state now with the knowledge of both $l_{i}$ and $l_{i}^{(\mathrm{c})}$, and assuming $l_{i} \leq l_{\max }^{(\mathrm{b})}+l_{\max }^{(\mathrm{c})}$ is always satisfied, we can deduce $l_{i}^{(\mathrm{b})}$ and accordingly $p_{i}^{(\mathrm{b})}$, in addition to the dropping $d_{i}$ given the relation $l_{i}=l_{i}^{(\mathrm{b})}+l_{i}^{(\mathrm{c})}+d_{i}$, and this completes the sketch of the proof.

In this study, we adopt SARSA algorithm which has the advantage of being online in a fully incremental fashion [16]. This algorithm is proper for problems having continuing tasks. In SARSA, the Q-function is updated as:

$$
\begin{aligned}
& Q\left(s_{i+1}, a_{i+1}\right) \leftarrow \\
& Q\left(s_{i}, a_{i}\right)+\sigma\left[R_{i+1}+\gamma Q\left(s_{i+1}, a_{i+1}\right)-Q\left(s_{i}, a_{i}\right)\right]
\end{aligned}
$$

where $R_{i+1}=\bar{r}_{i+1}$ is the instantaneous reward at time instant $i$, while $\gamma$ and $\sigma$ are the discount factor and learning rate, respectively. In [16], it has been proven that for any fixed policy SARSA, Q converges to optimal values with probability 1 if the learning rate (step-size) parameter satisfies the following conditions: $0 \leq \sigma_{i}<1, \sum_{0}^{\infty} \sigma_{i}=\infty$, and $\sum_{0}^{\infty} \sigma_{i}^{2}<\infty$. 


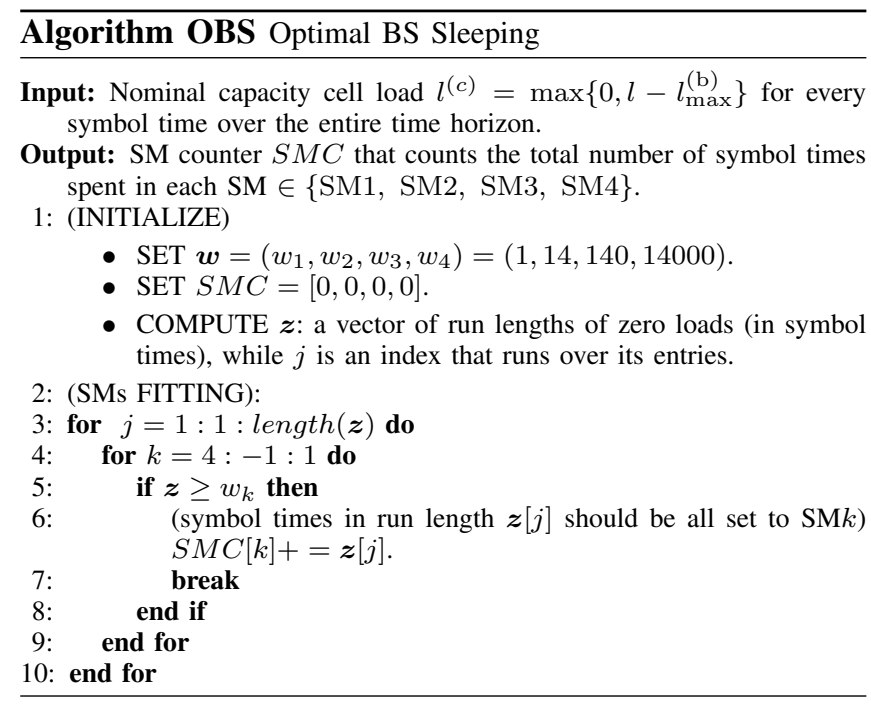

\section{E. Benchmarks}

We compare the performance of the proposed solution with (non-causal) optimal BS sleeping (OBS) as an upper bound on energy saving gain, and (non-adaptive) fixed SMs as a lower bound. OBS can be easily shown to be always feasible and optimal, although non-causal due to future information, thus marking an upper bound on energy saving. Also, OTABS only requires local information/counters, i.e., number of instantaneously requested PRBs at the physical site, which hosts both coverage and capacity cells. Exchanging timely information between distant sites also remains possible through tightly synchronized X2 interfaces.

1) Optimal BS Sleeping: In this scenario, the agent is fully aware of all future incoming PRBs and hence has the information of BS inactivity time. Knowing this inactivity period, the agent simply starts filling them from the deepest SM i.e., SM4, scanning all inactivity periods, and checking whether the wide SM4 duration can be accommodated. In the rest of unfilled inactivity period, we follow the same procedure for the next SM. Algorithm OBS explains the steps of this procedure which guarantees the highest energy saving gain, yet utilizing non-causal future knowledge.

2) Fixed SMs : In this scenario, we assume that during the inactive mode only SM1 or only SM2 can be used. It is worth noting that in the baselines all users are instantaneously served, hence no delay/dropping is incurred in the system.

\section{NUMERICAL RESULTS}

An event-based numerical simulation is implemented in Python, running the proposed adaptive algorithm and its baseline counterparts. The simulation parameters are defined in Table II. Fig. 1 shows the Q-function heatmap for normal hour, i.e., when the network is not crowded, and busy hour, i.e, when the network is in peak usage. Darker color means that this state-action pair is more frequently incurred. As defined, a state jointly specifies the SM and load level. In Fig. 1, we feed data of two hours, i.e., one busy and one normal hour, to the OTABS algorithm. The figure shows that the implemented Q-table can quickly adapt to the traffic pattern change, and manifests the agent's learned policy. The best policy in busy hours, as expected, is to stay in FM. If the BS is found in a deep SM, the agent selects to depart towards shallower SMs. This is intuitive since in busy hours
TABLE II: Studied System Configuration Parameters

\begin{tabular}{|c|c|c|}
\hline \multicolumn{2}{|c|}{ Parameter } & Value \\
\hline \multicolumn{2}{|c|}{ Modulation } & 16-QAM ( 4 bits per RE), $M=16$ \\
\hline \multicolumn{2}{|c|}{ Antennas } & 1 per cell sector, one sector per cell \\
\hline \multicolumn{2}{|c|}{ Bandwidth } & $5 \mathrm{MHz}$ for each (coverage and capacity) \\
\hline \multicolumn{2}{|c|}{ RBs per band } & $\begin{array}{c}25 \text { per } 5 \mathrm{MHz} \text { (LTE-A Compatible) } \\
\text { each RB spans } 12 \text { sub-carriers }\end{array}$ \\
\hline \multicolumn{2}{|c|}{ REs per TTI } & 14 (Based on FDD Frame Structure 1 [17]) \\
\hline \multicolumn{2}{|c|}{ Symbol Time } & $T_{\mathrm{s}}=\frac{1}{14 \times 1000}$ sec $\approx 72 \mu \mathrm{sec}$ \\
\hline \multirow{3}{*}{ SARSA } & $\sigma$ & 0.9 (learning rate) \\
\hline & $\gamma$ & 0.1 (discount factor) \\
\hline & $\epsilon$ & 0.9 (exploration parameter) \\
\hline
\end{tabular}

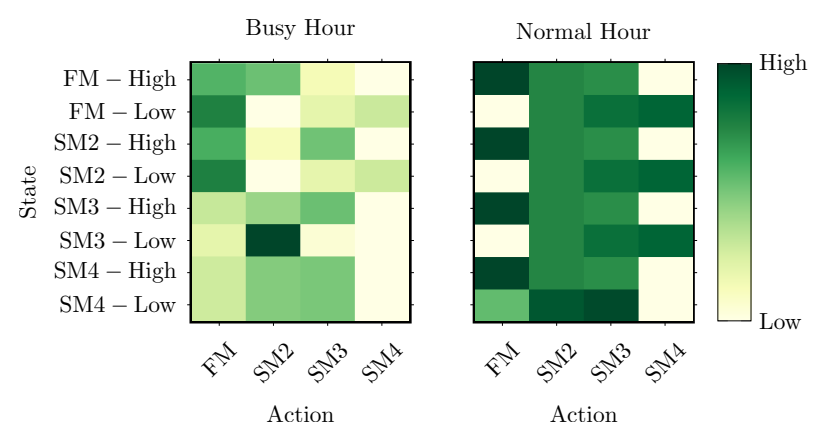

Fig. 1: Q-table heatmap for busy and normal hour. Darker stateaction pair color implies that the learnt policy takes this action with higher probability in the corresponding state.

it is highly likely to have frequent service requests, while being in deep SMs would incur high service interruption and accordingly packet drop rate. At normal hour, and if the load is low, the best policy is to select SM2 or SM3 to benefit from lower energy consumption of these SMs knowing that at normal hour it is less probable to have service requests. If load is found to be high, choosing FM remains to be the best policy while the chance of revisiting SM2 and SM3 is higher than that in busy hour.

Fig. 2 depicts the Q-table heatmap and learned policy as a function of the QoS trade-off factor $\alpha$. If $\alpha$ is close to 1 , more emphasis is given to preserve QoS compared to energy saving. On the contrary, when $\alpha$ approaches 0 , the agent tends to choose deeper SMs whenever possible for higher energy saving. For instance, when $\alpha=0.8$, and the current state is FM - Low, the agent still prefers to choose SM1 to avoid incurring any dropping. For the same state when $\alpha=0.2$, the agent prefers to choose either SM2 or SM3 since energy saving is given higher weight. Therefore, dynamically setting $\alpha$ can balance between energy saving and preserving QoS in different hours of the day. For instance, $\alpha$ closer to 1 avoids high dropping rate and is suitable for peak usage hours, while getting closer to 0 saves more energy which is proper for low load hours, e.g., at night. Simple regression can also map the desired licensespecified operator's dropping rate to the corresponding value of $\alpha$ for different load profiles.

In Fig. 3, we evaluate the energy saving/delay performance of OTABS against the two baselines. By increasing the dropping weight, the agent is more conservative in choosing deeper BS SMs, i.e., preferring shallower SMs. Clearly, this incurs less service delay, yet less energy saving. It can be also seen that OTABS achieves near optimal energy saving for $\alpha \leq 0.4$ where the selected actions are dominated by SM3 and SM4. 

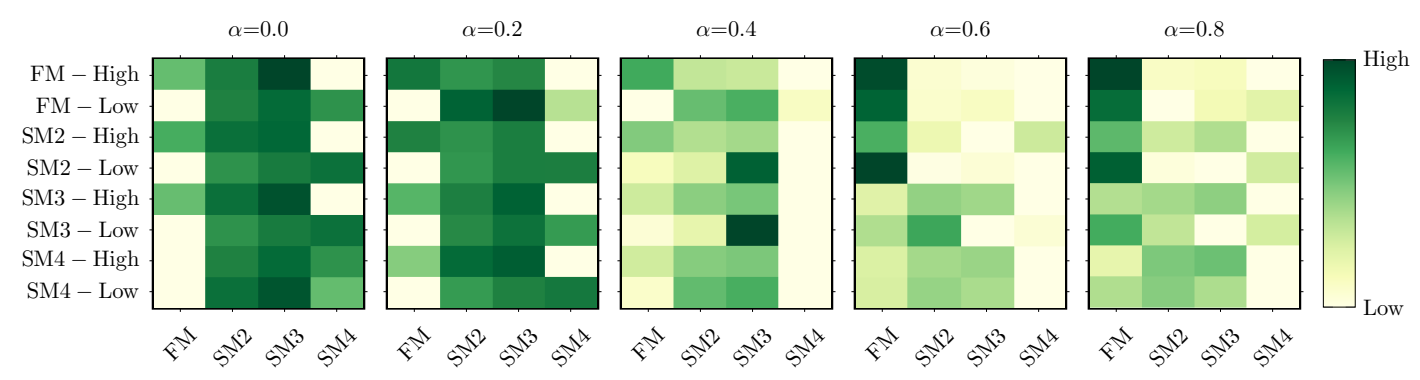

Fig. 2: Heatmap of Q-table for different energy saving-delay weights in the reward function.

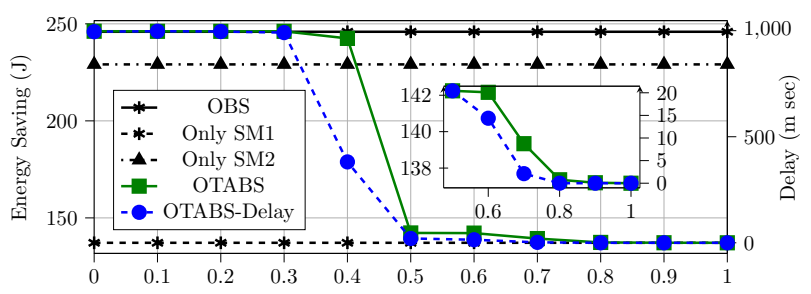

\section{REFERENCES}

[1] Masoudi et al., "Green mobile networks for 5G and beyond," IEEE Access, vol. 7, pp. 107 270-107 299, 2019.

[2] Olsson et al., "5GrEEn: Towards green 5G mobile networks," in Proc. IEEE WiMob'13. IEEE, 2013, pp. 212-216.

[3] ETSI, "Energy consumption and $\mathrm{CO} 2$ footprint of wireless networks," Report RRS05-024, 2011.

[4] Debaillie et al., "A flexible and future-proof power model for cellular base stations," in Proc. IEEE VTC-Spring'15, May 2015, pp. 1-7.

[5] Andersson et al., "Energy efficient heterogeneous network deployment with cell DTX," in Proc. IEEE ICC'16. IEEE, 2016, pp. 1-6.

[6] Piunti et al., "Energy efficient adaptive cellular network configuration with QoS guarantee," in Proc. IEEE ICC'15. IEEE, 2015, pp. 68-73.

[7] Elayoubi et al., "Optimal control for base station sleep mode in energy efficient radio access networks," in Proc. IEEE INFOCOM'11. IEEE, 2011, pp. 106-110.

Fig. 3: Energy saving, delay, and percentage of SMs for different reward weights.

\section{CONCLUSION}

We study energy saving in $5 \mathrm{G}$ base stations (BSs) by implementing multi-level sleep modes (SMs), where 4 different SMs are considered with their distinct (de)activation time and energy consumption. The studied scenario comprises a coverage cell for carrying $5 \mathrm{G}$ BS signaling and basic coverage in low-load time, and a capacity cell for activation in high-load times. We employ an online reinforcement learning (RL) algorithm to timely decide on the best BS SM at inactivity times, where the RL agent is rewarded for energy saving and penalized for packet dropping. A tradeoff is thus investigated between energy saving and incurred dropping which is controlled by the designed reward function. We utilize real network data obtained from a BS in Stockholm. Load data is first distilled in the form of number of requested resource blocks per time slot, then fed to the $\mathrm{RL}$ algorithm. The resulting energy saving and dropping performance is compared to two baselines; 1) non-causal optimal power saving with full knowledge of load over the entire time horizon, and 2) fixed (non-adaptive) BS sleeping. Results show that significant energy saving can be achieved if energy consumption holds the operator's utmost concern, while operating on batteries for instance, although at a cost of bearing higher dropping rate. Alternatively, when strict service quality measures are sought, the agent only selects the most shallow/fast-switching SMs to avoid any service interruption.

\section{ACKNOWLEDGMENT}

The authors would like to thank Luc Meunier and Anders Björk from Tele2 AB for their support with aggregated network traffic. This work is funded by EU Celtic-Next project AI4Green: Artificial Intelligence for Green Networks.

[8] Tran et al., "Practical evaluation of on-demand small cell on/off based on traffic model for 5G cellular networks," in Proc. IEEE WCNC'16. IEEE, 2016.

[9] Sriram et al., "Joint functional splitting and content placement for green hybrid CRAN," in Proc. IEEE PIMRC'19 2019. IEEE, 2019, pp. 1-7.

[10] Alabbasi et al., "Optimal processing allocation to minimize energy and bandwidth consumption in hybrid CRAN," IEEE Transactions on Green Communications and Networking, vol. 2, no. 2, pp. 545-555, 2018.

[11] 3GPP, "Radio resource control (RRC) protocol specification," Report V15.1.0, 2018.

[12] Salem et al., "Advanced sleep modes and their impact on flow-level performance of 5G networks," in Proc. IEEE VTC-Fall'17, Sep. 2017.

[13] _ _ "Reinforcement learning approach for advanced sleep modes management in 5G networks," in Proc. IEEE VTC-Fall'18, Chicago, USA, Aug. 2017.

[14] _ - "Traffic-aware advanced sleep modes management in 5G networks," in Proc. IEEE WCNC'19, Marrakech, Morocco, Apr. 2019.

[15] Pervaiz et al., "Energy-efficient and load-proportional eNodeB for 5G user-centric networks: A multilevel sleep strategy mechanism," IEEE Vehicular Technology Magazine, vol. 13, no. 4, pp. 51-59, 2018.

[16] Sutton and Barto, Reinforcement learning: An introduction. MIT press, 2018.

[17] Cox, An Introduction to LTE: LTE, LTE-Advanced, SAE, VoLTE and $4 G$ Mobile Communications, 2nd ed. Wiley Publishing, 2014.

[18] imec, GreenTouch Project, "Power model for wireless base stations." [Online]. Available: https://www.imec-int.com/en/powermodel

[19] GreenTouch Foundation, "GreenTouch final results from Green Meter research study,” White Paper V1.0, Jun. 2015. 